Background Young women in South Africa are highly affected by sexually transmitted infections (STI), like C. trachomatis (CT) and N. gonorrhoeae (NG). We aimed to estimate the incidence of CT and NG, and its determinants, among young women from the Western Cape, South Africa, participating in an HPV vaccine trial (the EVRI study).

Methods HIV-negative women aged 16-24 years were enrolled between October 2012 and July 2013. At enrolment and month 6 participants were screened for CT and NG. A questionnaire on demographic and sexual history characteristics was completed at enrolment and month 7. Treatment for CT and/or NG was offered to infected participants. Incidence rates (IR) of CT and NG were estimated. Determinants of incident CT and NG infections were assessed using Poisson regression.

Results 365 women were tested for CT and/or NG at least twice. Prevalence of CT and NG at baseline was 33.7\% and $10.4 \%$, respectively. Prevalence of co-infection with CT and NG was 7.1\%. During 113.3 person-years (py), 48 incident CT infections were diagnosed ( $\mathrm{IR}=42.4$ per 100 py, 95\% confidence interval (CI) 31.9-56.2). Twenty-nine incident NG were diagnosed during 139.3 py (IR=20.8 per 100 py, 95\%CI 14.5-29.9). Prevalent CT infection at baseline was associated with incident CT (adjusted incidence rate ratio (aIRR) 5.8, 95\%CI 3.0-11.23. More than three lifetime sex partners increased the risk for incident NG (3-4 partners aIRR $=7.3,95 \%$ CI 2.1-26.0; $\geq 5$ partners aIRR $=4.3,95 \% \mathrm{CI}$ 1.1-17.5).

Conclusions The IR of bacterial STIs among young women in the Western Cape is very high. Besides being previously infected and a higher lifetime number of sex partners, no other risk factors were found for CT and NG, suggesting that all these women were at risk. This indicates the need for intensified prevention of STIs as well as screening and treatment programs to increase sexual health in this region.

\section{P097 A MIXED-METHOD APPROACH TO MAPPING HEMATOLOGISTS' HIV TESTING BEHAVIOR IN LYMPHOMA PATIENTS AT THE AMSTERDAM UNIVERSITY MEDICAL CENTERS}

${ }^{1} \mathrm{~A}$ Ndong*, ${ }^{*} \mathrm{~S}$ Bogers, ${ }^{2} \mathrm{M}$ Kersten, ${ }^{3} \mathrm{P}$ Reiss, ${ }^{1} \mathrm{~S}$ Geerlings. 'Department of infectious diseases, Amsterdam University Medical Centers and Amsterdam Infection and Immunity Institute, Amsterdam, The Netherlands; ${ }^{2}$ Department of hematology, Amsterdam University Medical Centers, The Netherlands, Amsterdam, The Netherlands; ${ }^{3}$ Department of Global Health, Amsterdam University Medical Centers, Amsterdam Institute for Global Health and Development, and HIV Monitoring Foundation, Amsterdam, The Netherlands, Amsterdam, The Netherlands

\subsection{6/sextrans-2021-sti.226}

Background Compared to HIV-negative individuals, people living with HIV have a higher risk of malignant lymphoma. To what extent hematologists miss HIV testing opportunities among lymphoma patients (LP) in the Amsterdam University Medical Centers (UMC) is currently unknown. We quantified the HIV testing rate among LP and assessed factors influencing hematologists' HIV testing behavior in LP at both Amsterdam UMC locations.

Methods In this mixed-methods study, quantitative data from 2015 to 2019 from electronic health records of LP were retrospectively collected to assess both the HIV testing rate within three months around lymphoma diagnosis and the HIV positivity prevalence. An online survey among hematologists and semi-structured interviews among hematologists and authors of hematology guidelines were conducted.

Results Data from 656 LP and 21 hematologists who responded to the survey out of 40 were used. Interviews were held with four hematologists and two authors of hematology guidelines. The HIV testing rates were $56.4 \%$ and $57.6 \%$, and the HIV positivity prevalences were $0.8 \%$ and $0.9 \%$ for both locations. Eighty-five percent (18/21) of hematologists indicated they often offered an HIV test to LP in the past year, and 42\% (9/21) revealed HIV testing in LP is often discussed in their department. The type of lymphoma, lack of awareness regarding HIV testing recommendations in LP, low perceived risk of HIV among LP because of older age, and lack of guidelines recommending universal HIV testing regardless of lymphoma type, each influenced hematologists' HIV testing behavior.

Conclusion Our results show routine HIV testing among LP and hematologists' attitudes and knowledge towards testing at the Amsterdam UMC are suboptimal, despite HIV testing being recommended in most lymphoma guidelines. This highlights the need for interventions to improve hematologists' behavior towards testing, such as education, as well as expansion and enhanced implementation of the hematology guidelines.

\section{P098 A CAPITE AD CALCEM APPROACH IMPORTANCE IN CASE OF SYPHILIS}

${ }^{1,2} \mathrm{~A}$ Kalva*, 'V Bondare-Ansberga, 1,2 I Hartmane, 1,21 Mikazans. 'Rigas 1st hospital, Riga, Latvia; ${ }^{2}$ Riga Stradins University, Riga, Latvia

\subsection{6/sextrans-2021-sti.227}

Background Nowadays, the patient full examination is challenging by limited time in different medical fields (Musellim B. et al, 2017). It can lead inappropriate diagnosis and treatment. Therefore, it is important to use patient examination gold Approach a capite ad calcem.

Methods The medical documentation, pathology slides and data of laboratory results were reviewed in the context of upto-date medical literature.

Results A 34-year-old white man presents with a complaint about itching on his legs and arms that follow him around four months.

In objective examination of the overall status - without any deviation of the norm. In the local status, we found infiltrative, hyperaemic papules with yellowish crusted erosions on both legs and arms with well demarcated. On both legs we found varicose veins. Sexual history said that last sexual contact had been two years ago. The full blood test, infection tests (HIV, RPR, TPHA, HCV), microbiology and skin punch biopsy were done. Work diagnosis at the beginning: exacerbation of chronic eczema. Important to keep in mind, cutaneous features of secondary syphilis sometime can show as chronic dermatitis.

The blood test result, total IgE showed without any deviation of the norm. RPR - positive $(4+)$, TPHA $(4+, 1: 1280)$, HIV - negative, HCV - negative. Excreted from the skin: Staphylococcus aureus, Streptococcus pyogenes (beta haemolytic group A). Other sexual transmissive disease - negative. The skin punch biopsy was done from arm skin. At the pathophysiological conclusion said that: histological picture is 
possible in the case of Lyme disease. Lyme disease parameters are not determined due to treatment interruption at the patient's request.

Conclusion A capite ad calcem approach is an essential part in dermatovenerology practice. It can help for doctor find not just seemingly the main reason for patient's complain but allow watch wider.

\section{P099 HIGH PREVALENCE OF CURABLE SEXUALLY TRANSMITTED INFECTIONS AMONG HIV-UNINFECTED WOMEN PLANNING FOR PREGNANCY IN KWAZULU- NATAL, SOUTH AFRICA}

${ }^{1}$ I Beesham*, ${ }^{1} \mathrm{~S}$ Bosman, ${ }^{1} \mathrm{M}$ Jaggernath, ${ }^{1} \mathrm{Y}$ Kriel, ${ }^{2} \mathrm{P}$ Chitneni, ${ }^{3} \mathrm{P}$ Smith, ${ }^{4} \mathrm{~K}$ Bennett, 1J Smit, ${ }^{3,5} \mathrm{~L}$ Matthews. ${ }^{1} \mathrm{MRU}$ (MatCH Research Unit), Faculty of Health Sciences, University of Witwatersrand, Durban, South Africa; ${ }^{2}$ Brigham and Women's Hospital, Massachusetts General Hospital Center for Global Health, Boston, USA; ${ }^{3}$ University of Alabama at Birmingham, School of Medicine, Division of Infectious Diseases, Birmingham, USA; ${ }^{4}$ Bennett Statistical Consulting, Ballston Lake, USA; ${ }^{5}$ Division of Infectious Disease, Massachusetts General Hospital, Boston, USA

\subsection{6/sextrans-2021-sti.228}

Background HIV-exposed women in African settings planning to conceive are at heightened risk of acquiring sexually transmitted infections (STIs). STIs can increase HIV acquisition risks and cause maternal and child morbidity. Here, we describe the prevalence of curable STIs among HIV-uninfected women planning pregnancy with a partner who is living with HIV or of unknown serostatus in an HIV-endemic setting (eThekwini, KwaZulu-Natal (KZN)).

Methods This is a descriptive longitudinal sub-study nested within the Safer Conception for Women study. HIV-uninfected women, aged 18-35 years with personal or partner plans for pregnancy were enrolled from November 2019 in eThekwini, KZN. A questionnaire was administered, and women were examined for STIs, and tested for Chlamydia trachomatis (CT), Neisseria gonorrhoeae (NG), Trichomonas vaginalis (TV), and Mycoplasma genitalium (MG) via PCR of a provider-collected swab, and Treponema Pallidum (TP) via TPHA testing. Women with symptomatic and/or laboratory-confirmed STIs are treated and provided with partner notification letters. Repeat sample collection is scheduled after six months, and during pregnancy. Here, we report on prevalent curable STIs at baseline.

Results Among the 50 women enrolled, [median age: 24 (range: 19-33), HIV serostatus of partner: 47(94\%) unknown], $3(6 \%)$ reported STI symptoms, and 12 (24\%) had curable STI organisms detected on laboratory testing. CT was the most frequent pathogen $(6,12 \%)$, followed by TV $(5$, $10 \%)$, NG (2, 4\%) and MG (1, 2\%). Two women had >1 STI pathogen. No TP was detected. Baseline demographic and sexual behavioural characteristics did not differ between women with and without STIs.

Conclusion Our findings indicate a high prevalence of curable STIs, mostly asymptomatic, among HIV-uninfected women planning for a pregnancy in the next year. Given the impact of asymptomatic curable STIs on maternal and neonatal health, this population should be prioritized for objective STI testing.

\section{P100 \\ HIV TESTING AND ASSOCIATED FACTORS IN MEN WHO HAVE SEX WITH MEN BY URBANIZATION-LEVEL: A CROSS-SECTIONAL STUDY IN THE NETHERLANDS}

${ }^{1,2} \mathrm{~J}$ Leenen, ${ }^{1,2} \mathrm{~J}$ Wijers, ${ }^{3,4,5} \mathrm{C}$ den Daas, ${ }^{5,6} \mathrm{~J}$ de Wit, ${ }^{1,2,7} \mathrm{C}$ Hoebe, ${ }^{1,8} \mathrm{~N}$ Dukers-Muijrers ${ }^{*}$ ${ }^{1}$ Department of Sexual Health, Infectious Diseases and Environmental Health, South Limburg, Heerlen, The Neterlands; ${ }^{2}$ Public Health Service (GGD Zuid Limburg), Heerlen, The Netherlands; ${ }^{3}$ Department of Medical Microbiology, Care and Public Health Research Institute (CAPHRI), Maastricht University Medical Centre (MUMC+), Maastricht, The Netherlands; ${ }^{4}$ Aberdeen Health Psychology Group, Institute of Applied Health Sciences, University of Aberdeen, Aberdeen, UK; ${ }^{5}$ Center for Infectious Disease Control, National Institute for Public Health and the Environment, Bilthoven, The Netherlands; ${ }^{6}$ Department of Interdisciplinary Social Science, Faculty of Social and Behavioral Sciences, Utrecht University, Utrecht, The Netherlands; ${ }^{7}$ Centre for Social Research in Health, University of New South Wales, Sydney, Australia; ${ }^{8}$ Department of Social Medicine, Care and Public Health Research Institute (CAPHRI), Maastricht University Medical Centre $(M U M C+)$, Maastricht, The Netherlands

\subsection{6/sextrans-2021-sti.229}

Background Testing is a key strategy towards elimination of HIV; preferably by regional targeted approaches. Regular HIV testing enables timely care and reduces HIV transmission. We assessed HIV-testing behaviour in men who have sex with men (MSM), and factors associated with never and not recent testing by urbanisation-level.

Methods The online 'Men \& Sexuality' survey was conducted in 3,815 MSM (mean age 36 years; the Netherlands, 2018). HIV-testing was defined as recent (<1year), not recent ( $\geq 1$ year), and never. Testing was evaluated as outcome (recent testing was reference) in multivariable multinominal regression analyses (calculating odds ratio's [ORs]). We evaluated associations with urbanization-level. We also evaluated associations with a range of sociodemographic/behavioral factors in analyses stratified by urbanization-level (high-urban [ $>2,500$ living addresses $/ \mathrm{km} 2]$ and non-high-urban $[\leq 2,500$ living addresses/ $\mathrm{km} 2]]$ ).

Results In high-urban areas, 68.4\% of MSM, and in non-highurban areas, 54.9\%, recently tested; $11.8 \%$ in high-urban and $25.2 \%$ in non-high-urban areas, never tested (aOR: 1.89; $1.55-2.31, \mathrm{p}<0.001)$.

In high-urban areas: factors associated with never and not recent testing were younger age, fewer new sex partners, never notified for HIV, no condomless anal intercourse, and self-identification as bisexual (versus gay).

In non-high-urban areas: additionally associated were moderate/low perceived HIV severity, moderate/low HIV risk perception, and moderate/low share of MSM among friends (versus 'high'). In non-high-urban areas 27\% reported a high share of MSM among friends (high-urban: 38\%).

In non-high-urban areas, never tested MSM would prefer self-sampling/self-testing (high-urban: facility-based testing).

Conclusion The proportion of MSM who never tested was high $(25 \%)$ in non-high-urban areas in The Netherlands; twofold compared to high-urban areas. In non-high urban areas, MSM who have a more restricted MSM-defined social context, may be less likely to test, and may benefit from targeted outreach and test-barrier-reducing strategies, e.g. by self-testing/self-sampling testing. A regional approach, accounting for urbanization-related factors, to eliminate HIV is warranted. 